Research Article

\title{
The Method of Fischer-Riesz Equations for Elliptic Boundary Value Problems
}

\author{
A. Alsaedy and N. Tarkhanov \\ Institut für Mathematik, Universität Potsdam, Am Neuen Palais 10, 14469 Potsdam, Germany \\ Correspondence should be addressed to A. Alsaedy; alsaedy@math.uni-potsdam.de and N. Tarkhanov; tarkhanov@math.uni- \\ potsdam.de
}

Received 22 October 2012; Accepted 30 December 2012

Academic Editor: Vladislav Kravchenko

Copyright (C) 2013 A. Alsaedy and N. Tarkhanov. This is an open access article distributed under the Creative Commons Attribution License, which permits unrestricted use, distribution, and reproduction in any medium, provided the original work is properly cited.

We develop the method of Fischer-Riesz equations for general boundary value problems elliptic in the sense of Douglis-Nirenberg. To this end we reduce them to a boundary problem for a (possibly overdetermined) first-order system whose classical symbol has a left inverse. For such a problem there is a uniquely determined boundary value problem which is adjoint to the given one with respect to the Green formula. On using a well-elaborated theory of approximation by solutions of the adjoint problem, we find the Cauchy data of solutions of our problem.

\section{Introduction}

The aim of this paper is to bring together two areas in which integral formulas like Green's formula for harmonic functions are of great importance. The first area is complex analysis where the method of integral representations was a central tool over the second half of the 20th century, see [1]. And the second area is the theory of elliptic boundary problems where the parametrix method led to the most refined results, such as local principle, $C^{*}$-algebras of pseudodifferential boundary value problems [2], and so forth.

The method of Fischer-Riesz equations can be specified within a larger approach which is usually referred to as the boundary element method. By this latter is meant a numerical method of solving boundary value problems which have been formulated as boundary integral equations. It can be applied in many areas of engineering and science including fluid mechanics, acoustics, electromagnetics, and fracture mechanics, see [3-6].

The boundary elements method attempts to use the given boundary conditions and other data of the problem to fit boundary values into the integral equation, rather than values throughout the space defined by a system of partial differential equations. Once this is done, the boundary integral equation can be used again to calculate numerically the solution directly at any desired point in the solution domain. More precisely, from the Cauchy data of the solution on the whole boundary one calculates readily the solution in the domain provided a left fundamental solution of the system is available in an explicit form, see for instance Lemma 10.2.3 in [7].

The idea of the method of Fischer-Riesz equations goes back at least as far as [8]. The paper [9] was given by Picone as an invited address before the Second Austrian Mathematical Congress in Insbruck in 1949. He states in the introduction that he asked Fichera to write a certain part of the report. It is a crystallisation in the form of an abstract theory of some of the methods used by the authors and their associates at the National Institute for Applied Mathematics in Rome in the solution of problems involving differential and integro-differential equations. The method is based on some functional interpretation of the relations of mathematical physics analogous to Green's formula. The central point of the method is a construction of a suitable sequence of functions which are complete in a Lebesgue space $L^{2}$ on the boundary and satisfy the formal adjoint system in a neighbourhood of the closure of the domain. In [9] there were no indication to any solution of this problem. In [10] a general process of constructing a necessary complete sequence of functions was elaborated assuming an explicit fundamental solution of the system. However, this paper fell short of providing a functiontheoretic description of the method. In [11] the second author 
published a proof of a theorem of functional analysis that had already been obtained at the end of the 1980s. As it became clear later, this theorem was just an abstract exposition of the Fischer-Riesz equations method mentioned in [10]. In [11] this method was developed for studying the ill-posed Cauchy problem with data on a piece of the boundary for solutions of overdetermined elliptic systems, see also Chapter 11 in [7].

The purpose of the present paper is to develop the method of Fischer-Riesz equations for general boundary value problems for systems of partial differential equations elliptic in the sense of Douglis-Nirenberg [12]. To escape technicalities related to assigning weights we exploit the result of [13] and reduce the system to a (possibly overdetermined) first-order system whose classical symbol has a left inverse away from the zero section of the cotangent bundle. In this way we obtain what is often referred to as the first order system with injective symbol. The advantage of such systems lies in the fact that the Cauchy data of a solution just amount to the restriction of the solution to the boundary of the domain. Moreover, to any first order system there corresponds a unique Green operator which leads to a canonical Green formula for solutions. Any normal boundary conditions for solutions of the source system then reduce to an inhomogeneous linear system in the space of Cauchy data.

In contrast to [11] we elaborate the method of FischerRiesz equations for elliptic boundary value problems in Sobolev spaces, for these latter fit well the Fredholm property. If the boundary value problem is Fredholm, then the conditions of solvability obtained by the Fischer-Riesz equations method come to those obtained from the Fredholm theory, that is, the orthogonality to solutions of the homogeneous boundary value problem adjoint relative to the Green formula. The method of Fischer-Riesz equations may then be developed as a tool to get effective approximate solutions, $\mathrm{cf}$. [10].

\section{Reduction to a First-Order System}

One of the fundamental problems in the theory of partial differential equations is the problem of classifying equations and systems by type. A specific problem associated with the definition of ellipticity is that when a higher-order equation or system is reduced to a first order system, ellipticity may be destroyed. (We manipulate the concept "elliptic" freely. This concept can be given a strong sense only in an operator algebra with symbol map, where by the ellipticity of an operator is meant the invertibility of its symbol.) One approach to this problem was introduced in the paper [12] which gave a definition of ellipticity for systems which involved assigning weights to each of the equations and dependent variables and then defining the principal part of the system in terms of those weights. This concept can also be interpreted in terms of generalised homogeneity based on certain group actions in the spaces of preimages and images, see equality (1.4) in [14]. The advantage of the definition of ellipticity given in [12] is that ellipticity can be preserved, while a higher-order equation or system is reduced to an equivalent first order system. The disadvantage is that the definition is not invariant under nonsingular changes of variables. Therefore, the approach via weights fails to properly recognise elliptic systems. An alternative approach suggested in [13] is to reduce the original equation or system to an overdetermined first order system and then use the classical symbol, which is natural and invariant way for such systems. In [15] this result is strengthened by showing that any determined or overdetermined system with smooth coefficients and injective Douglis-Nirenberg symbol can be reduced to an overdetermined first order system with smooth coefficients and injective classical symbol. This reduction is accomplished by introducing as new dependent variables, the derivatives of some of the original variables, and adjoining equations describing the relations between the new variables and the old or among the new variables. Moreover, any overdetermined first order system with smooth coefficients and injective classical symbol can be converted to a determined second-order systems which is elliptic in the sense of Douglis-Nirenberg or under any reasonable definition of ellipticity. The conversion is accomplished by operating on the original system with an appropriately chosen first-order operator. The conversion to a second order system allows the application of the regularity results of [16]. In fact, second order systems are treated in detail in [16]. Note that the systems of partial differential equations usually still contain hidden integrability conditions. The process of their explicit construction is called completion (to involution). In [17] it is shown that the completion of any determined or overdetermined system with injective Douglis-Nirenberg symbol leads to an equivalent system whose classical symbol has a left inverse. To formulate the main result of [15] more precisely, we extend the concept of ellipticity in the sense of Douglis-Nirenberg to overdetermined systems.

The systems we consider are of the form

$$
A=\left(A_{i, j}\right)_{\substack{i=1, \ldots, l \\ j=1, \ldots, k}},
$$

where $A_{i, j}$ are scalar partial differential operators of order $m_{i, j}$ on an open set $\mathscr{X}$ in $\mathbb{R}^{n}$ and $l \geq k$. (We will generally use the convention that our source system has $k$ dependent variables, $l$ equations, and $n$ independent variables.)

Definition 1. Suppose there are weights $s_{1}, \ldots, s_{l}$ and $t_{1}, \ldots, t_{k}$ in $\mathbb{Z}$, such that $m_{i, j} \leq s_{i}+t_{j}$. With this structure, the principal symbol of (1) is the matrix

$$
\sigma_{\mathrm{DN}}(A)(x, \xi)=\left(\sigma^{s_{i}+t_{j}}\left(A_{i, j}\right)(x, \xi)\right)_{\substack{i=1, \ldots, l \\ j=1, \ldots, k}}
$$

for $(x, \xi) \in T^{*} \mathscr{X}$, where $\sigma^{s_{i}+t_{j}}\left(A_{i, j}\right)$ is the homogeneous component of degree $s_{i}+t_{j}$ of the full symbol of $A_{i, j}$.

System (1) is said to have injective symbol in the sense of Douglis-Nirenberg at $x_{0} \in \mathscr{X}$ if $\sigma_{\mathrm{DN}}(A)(x, \xi)$ has maximal rank (i.e., rank $k$ ) for $x=x_{0}$ and all $\xi \in \mathbb{R}^{n} \backslash\{0\}$.

The numbers $s_{1}, \ldots, s_{l}$ and $t_{1}, \ldots, t_{k}$ are determined uniquely up to an additive constant. Hence, the weights can be normalised by the condition $s_{1}, \ldots, s_{l} \leq 0$ and $\max s_{i}=0$. Then $t_{j}$ satisfy automatically $t_{1}, \ldots, t_{k} \geq 0$, for if $t_{j}<0$, then $s_{i}+t_{j}<0$, and so all the operators $A_{1, j}, \ldots, A_{l, j}$ vanish identically. This amounts to saying that the $j$ th dependent variable does not enter into the system, which is impossible. 
Let $\left(\kappa_{\lambda}\right)_{\lambda>0}$ and $\left(\widetilde{\kappa}_{\lambda}\right)_{\lambda>0}$ be the group actions in $\mathbb{C}^{k}$ and $\mathbb{C}^{l}$, respectively, given by

$$
\begin{aligned}
& \kappa_{\lambda}=\lambda^{|t|} \operatorname{diag}\left(\lambda^{-t_{1}}, \ldots, \lambda^{-t_{k}}\right), \\
& \widetilde{\kappa}_{\lambda}=\lambda^{-|s|} \operatorname{diag}\left(\lambda^{s_{1}}, \ldots, \lambda^{s_{l}}\right),
\end{aligned}
$$

where $|s|=s_{1}+\cdots+s_{l}$ and $|t|=t_{1}+\cdots+t_{k}$. Then the principal symbol $\sigma_{\mathrm{DN}}(A)$ is homogeneous of degree $|s|+|t|$ in the sense that

$$
\sigma_{\mathrm{DN}}(A)(x, \lambda \xi)=\lambda^{|s|+|t|} \widetilde{\kappa}_{\lambda} \sigma_{\mathrm{DN}}(A)(x, \xi) \kappa_{\lambda}^{-1}
$$

for all $\lambda>0$. For $s_{1}=\cdots=s_{l}=0$, one recovers the principal part of $A$ considered by I. G. Petrovskii. For $s_{1}=$ $\cdots=s_{l}=0$ and $t_{1}=\cdots=t_{k}=m$, one obtains the classical principal symbol. The so-called twisted homogeneity of type (4) is of great importance in the calculus of pseudodifferential operators with operator-valued symbols.

The following result is due to [15].

Theorem 2. Any system (1) with coefficients of class $C^{s, h}$ and injective symbol in the sense of Douglis-Nirenberg in $\mathscr{X}$ can be converted to an equivalent overdetermined first order system whose coefficients are of class $C^{s-1, h}$, and whose classical symbol is injective.

Proof. As is mentioned in [15], the reduction procedure used here is related to that attributed to Atiyah and Singer. It is probably not optimal in the sense that it may lead to a first order system which is not the smallest possible representation of the original system.

\section{Green Formula}

From what has been proved in Section 2 it follows that there is no restriction of generality in assuming that $A$ is a (possibly overdetermined) first order partial differential operator with injective symbol on an open set $\mathscr{X} \subset \mathbb{R}^{n}$. Thus, $A$ is of the form

$$
A(x, D)=\sum_{j=1}^{n} A_{j}(x) D_{j}+A_{0}(x),
$$

where $A_{1}(x), \ldots, A_{n}(x)$ and $A_{0}(x)$ are $(l \times k)$-matrices of smooth functions on $\mathscr{X}$ and $D_{j}=-\imath \partial_{x_{j}}$ with $\imath=\sqrt{-1}$. We require

$$
\sigma^{1}(A)(x, \xi):=\sum_{j=1}^{n} A_{j}(x) \xi_{j}
$$

to have maximal rank (i.e., $\operatorname{rank} k$ ) for all $(x, \xi)$ away from the zero section of $T^{*} \mathscr{X}$.

In order to get asymptotic results, it is necessary to put some restrictions on $A$. Our basic assumption is that $A$ satisfies the uniqueness condition of the local Cauchy problem in $\mathscr{X}$ (condition $(U)_{s}$, cf. [7, p. 185]). That is, if $u$ is a solution of $A u=0$ on a connected open set $U \subset \mathscr{X}$ and $u$ vanishes on a nonempty open subset of $U$, then $u \equiv 0$ in $U$.
Lemma 3. If $A$ satisfies the condition $(U)_{s}$ in $\mathscr{X}$, then it has a pseudodifferential left fundamental solution; that is, there is an $(k \times l)$-matrix $\Phi$ of classical pseudodifferential operators of order -1 on $\mathscr{X}$, such that $\Phi A=I$ on compactly supported distributions in $\mathscr{X}$ with values in $\mathbb{C}^{k}$.

Proof (see Theorem 4.4.3 of [7]). By the very construction, $\Phi$ has rational symbol; that is, it satisfies the transmission condition with respect to each hypersurface in $\mathscr{X}$.

Let $\mathscr{D}$ be a relatively compact domain with smooth boundary in $\mathscr{X}$ and $B$ any $\left(k^{\prime} \times k\right)$-matrix of smooth functions on the boundary $\partial \mathscr{D}$ of $\mathscr{D}$, such that the rank of $B(x)$ is equal to $k^{\prime}$ for all $x \in \partial \mathscr{X}$. We are interested in the boundary value problem

$$
\begin{array}{ll}
A u=f & \text { in } \mathscr{D}, \\
B u=u_{0} & \text { at } \partial \mathscr{D}
\end{array}
$$

with data $u_{0}$ on $\partial \mathscr{D}$. The most conventional Hilbert space setting of this problem is $H_{1}:=W^{1,2}$, hence we choose $u_{0}$ in $H^{1 / 2}\left(\partial \mathscr{D}, \mathbb{C}^{k^{\prime}}\right)$ and look for a $u \in H^{1}\left(\mathscr{D}, \mathbb{C}^{k}\right)$ satisfying (7).

Lemma 4. Let $C$ be $a\left(\left(k-k^{\prime}\right) \times k\right)$-matrix of smooth functions on $\partial \mathscr{D}$, such that

$$
\operatorname{rank}\left(\begin{array}{c}
B(x) \\
C(x)
\end{array}\right)=k
$$

for all $x \in \partial \mathscr{D}$. Then there are unique matrices $B^{*}$ and $C^{*}$ of continuous functions on $\partial \mathscr{D}$ with the property that

$$
\begin{array}{r}
\int_{\partial \mathscr{D}}\left(\left(B u, C^{*} g\right)_{x}-\left(C u, B^{*} g\right)_{x}\right) d s \\
=\int_{\mathscr{D}}\left((A u, g)_{x}-\left(u, A^{*} g\right)_{x}\right) d x
\end{array}
$$

for all $u \in H^{1}\left(\mathscr{D}, \mathbb{C}^{k}\right)$ and $g \in H^{1}\left(\mathscr{D}, \mathbb{C}^{l}\right)$, where $d s$ is the surface measure on the boundary.

As usual, we write $A^{*}$ for the formal adjoint of the differential operator $A$ on the open set $\mathscr{X}$.

Proof. By assumption, the $(k \times k)$-matrix

$$
T(x)=\left(\begin{array}{l}
B(x) \\
C(x)
\end{array}\right)
$$

is invertible for all $x \in \partial \mathscr{D}$. Write $(T(x))^{-1}=\left(T_{1}(x), T_{2}(x)\right)$ where $T_{1}$ and $T_{2}$ are $\left(k \times k^{\prime}\right)$ - and $\left(k \times\left(k-k^{\prime}\right)\right)$-matrices of smooth functions on $\partial \mathscr{D}$, respectively. The equalities $T^{-1} T=$ $E_{k}$ and $T T^{-1}=E_{k}$ amount to $T_{1} B+T_{2} C=E_{k}$ and

$$
\begin{gathered}
B T_{1}=E_{k^{\prime}}, \quad B T_{2}=0, \\
C T_{1}=0, \quad C T_{2}=E_{k-k^{\prime}},
\end{gathered}
$$

where $E_{k}$ stands for the unity $(k \times k)$-matrix. 
Given any $u \in H^{1}\left(\mathscr{D}, \mathbb{C}^{k}\right)$ and $g \in H^{1}\left(\mathscr{D}, \mathbb{C}^{l}\right)$, the Green formula of [7, Section 9.2.2] shows that

$$
\int_{\partial \mathscr{D}}(\sigma(x) u, g)_{x} d s=\int_{\mathscr{D}}\left((A u, g)_{x}-\left(u, A^{*} g\right)_{x}\right) d x
$$

where $\sigma(x)$ is the principal symbol of $A$ evaluated at the point $(x,-\imath v(x))$ of the complexified cotangent bundle of $X, v(x)$ being the outward normal unit vector of the boundary at $x \in$ $\partial \mathscr{D}$. Substituting $u=\left(T_{1} B+T_{2} C\right) u$ into this formula yields (9) with

$$
\begin{gathered}
C^{*}=\left(\sigma T_{1}\right)^{*}, \\
B^{*}=-\left(\sigma T_{2}\right)^{*},
\end{gathered}
$$

as desired.

From (13) it follows immediately that the rank of $C^{*}$ is equal to $k^{\prime}$ and the rank of $B^{*}$ is $k-k^{\prime}$.

Elliptic boundary value problems (7) require $k=l$ to be even and $k^{\prime}=k / 2$, in which case also the problem

$$
\begin{aligned}
& A^{*} g=v \text { in } \mathscr{D}, \\
& B^{*} g=g_{0} \text { at } \partial \mathscr{D}
\end{aligned}
$$

called adjoint to (7) with respect to the Green formula is actually elliptic, cf. [18].

Given any first order partial differential operators $A$ with injective symbol on $\mathscr{X}$, the composition $\Delta=A^{*} A$ is a second order elliptic operator in the classical sense. This operator is usually referred to as the Laplacian of $A$. An easy manipulation of Green formula (9) leads to a fairly structural Green formula for the Laplacian $\Delta$.

Theorem 5. Under the above notation, any functions $u, v \in$ $H^{2}\left(\mathscr{D}, \mathbb{C}^{k}\right)$ satisfy the integral equality

$$
\begin{gathered}
\int_{\partial \mathscr{D}}\left(\left(B u, C^{*} A v\right)_{x}-\left(C^{*} A u, B v\right)_{x}\right. \\
\left.-\left(C u, B^{*} A v\right)_{x}+\left(B^{*} A u, C v\right)_{x}\right) d s \\
=\int_{\mathscr{D}}\left((\Delta u, v)_{x}-(u, \Delta v)_{x}\right) d x .
\end{gathered}
$$

Proof. It suffices to apply (9) twice to the left-hand side of this equality. Cf. Corollary 9.2.12 of [7].

\section{Function Spaces}

Denote by $H^{1 / 2}\left(\mathscr{D}, \mathbb{C}^{k}\right)$ the Slobodetskii space of functions of fractional smoothness $1 / 2$ in $\mathscr{D}$ with values in $\mathbb{C}^{k}$, that is, the completion of $C^{\infty}\left(\overline{\mathscr{D}}, \mathbb{C}^{k}\right)$ with respect to the norm

$$
\begin{aligned}
& \|u\|_{H^{1 / 2}\left(\mathscr{D}, \mathbb{C}^{k}\right)} \\
& \quad=\left(\|u\|_{L^{2}\left(\mathscr{D}, \mathbb{C}^{k}\right)}^{2}+\iint_{\mathscr{D} \times \mathscr{D}} \frac{|u(x)-u(y)|^{2}}{|x-y|^{n+1}} d x d y\right)^{1 / 2} .
\end{aligned}
$$

Obviously, $H^{1 / 2}\left(\mathscr{D}, \mathbb{C}^{k}\right)$ is a Hilbert space. We use the continuous embedding $H^{1 / 2}\left(\mathscr{D}, \mathbb{C}^{l}\right) \hookrightarrow L^{2}\left(\mathscr{D}, \mathbb{C}^{l}\right)$ to specify the dual space of $H^{1 / 2}\left(\mathscr{D}, \mathbb{C}^{l}\right)$ via the pairing in $L^{2}\left(\mathscr{D}, \mathbb{C}^{l}\right)$. Namely, let $H^{-1 / 2}\left(\mathscr{D}, \mathbb{C}^{l}\right)$ be the completion of $C^{\infty}\left(\overline{\mathscr{D}}, \mathbb{C}^{l}\right)$ with respect to the norm

$$
\|f\|_{H^{-1 / 2}\left(\mathscr{D}, \mathbb{C}^{l}\right)}=\sup _{\substack{g \in C^{\infty}\left(\mathscr{D}, \mathbb{C}^{l}\right) \\ g \neq 0}} \frac{\left|(f, g)_{L^{2}\left(\mathscr{D}, \mathbb{C}^{l}\right)}\right|}{\|g\|_{H^{1 / 2}\left(\mathscr{D}, \mathbb{C}^{l}\right)}} .
$$

Using these spaces, we are in a position to enlarge the domain of problem (7). To this end, we write $\mathscr{H}^{1 / 2}\left(\mathscr{D}, \mathbb{C}^{k}\right)$ for the completion of $C^{\infty}\left(\overline{\mathscr{D}}, \mathbb{C}^{k}\right)$ with respect to the norm

$$
\|u\|_{\mathscr{H}^{1 / 2}\left(\mathscr{D}, \mathbb{C}^{k}\right)}=\left(\|u\|_{H^{1 / 2}\left(\mathscr{D}, \mathbb{C}^{k}\right)}^{2}+\|u\|_{L^{2}\left(\partial \mathscr{D}, \mathbb{C}^{k}\right)}^{2}\right)^{1 / 2} .
$$

By the trace theorem, the space $\mathscr{H}^{1 / 2}\left(\mathscr{D}, \mathbb{C}^{k}\right)$ contains any space $H^{s}\left(\mathscr{D}, \mathbb{C}^{k}\right)$ with $s>1 / 2$. However, the norm of $L^{2}\left(\partial \mathscr{D}, \mathbb{C}^{k}\right)$ is not majorised by the norm of $H^{1 / 2}\left(\mathscr{D}, \mathbb{C}^{k}\right)$.

Suppose $u \in \mathscr{H}^{1 / 2}\left(\mathscr{D}, \mathbb{C}^{k}\right)$ and $\left\{u_{j}\right\}$ is a sequence in $C^{\infty}\left(\overline{\mathscr{D}}, \mathbb{C}^{k}\right)$ converging to $u$ in the norm (18). Then $\left\{u_{j}\right\}$ is a Cauchy sequence in $H^{1 / 2}\left(\mathscr{D}, \mathbb{C}^{k}\right)$, and so it converges to an element $u_{i} \in H^{1 / 2}\left(\mathscr{D}, \mathbb{C}^{k}\right)$. Moreover, the restrictions of $u_{j}$ to the boundary form a Cauchy sequence in $L^{2}\left(\partial \mathscr{D}, \mathbb{C}^{k}\right)$. Hence, the sequence $\left.\left\{u_{j}\right\rceil_{\partial \mathscr{D}}\right\}$ converges in the space $L^{2}\left(\partial \mathscr{D}, \mathbb{C}^{k}\right)$ to an element $u_{b}$. It follows immediately that the closure of the mapping $u \mapsto\left(u\left\lceil_{\mathscr{D}}, u \uparrow_{\partial \mathscr{D}}\right)\right.$ is an isometry of $\mathscr{H}^{1 / 2}\left(\mathscr{D}, \mathbb{C}^{k}\right)$ onto a subspace of the Cartesian product $H^{1 / 2}\left(\mathscr{D}, \mathbb{C}^{k}\right) \times$ $L^{2}\left(\partial \mathscr{D}, \mathbb{C}^{k}\right)$. For this reason, each element $u \in \mathscr{H}^{1 / 2}\left(\mathscr{D}, \mathbb{C}^{k}\right)$ can be identified with its image $\left(u_{i}, u_{b}\right)$ in the Cartesian product. We call $u_{b} \in L^{2}\left(\partial \mathscr{D}, \mathbb{C}^{k}\right)$ the (generalised) trace of $u_{i} \in H^{1 / 2}\left(\mathscr{D}, \mathbb{C}^{k}\right)$ on the boundary of $\mathscr{D}$ in spite of the fact that the trace $u_{b}$ does not depend continuously on $u_{i}$.

Lemma 6. There is a constant $c>0$, such that

$$
\|A u\|_{H^{-1 / 2}\left(\mathscr{D}, \mathbb{C}^{l}\right)} \leq c\|u\|_{\mathscr{H}^{1 / 2}\left(\mathscr{D}, \mathbb{C}^{k}\right)}
$$

for all $u \in C^{\infty}\left(\overline{\mathscr{D}}, \mathbb{C}^{k}\right)$.

Proof. The proof is based on manipulations of Green formula (9). See Lemma 2.3.1 in [19].

It follows from Lemma 6 that the closure $A$ of the mapping $u \mapsto A u$ for $u \in C^{\infty}\left(\overline{\mathscr{D}}, \mathbb{C}^{k}\right)$ acts continuously from $\mathscr{H}^{1 / 2}\left(\mathscr{D}, \mathbb{C}^{k}\right)$ into $H^{-1 / 2}\left(\mathscr{D}, \mathbb{C}^{l}\right)$. Indeed, if $u \in \mathscr{H}^{1 / 2}\left(\mathscr{D}, \mathbb{C}^{k}\right)$ and $\left\{u_{j}\right\}$ is a sequence in $C^{\infty}\left(\overline{\mathscr{D}}, \mathbb{C}^{k}\right)$ converging to $u$ in $\mathscr{H}^{1 / 2}\left(\mathscr{D}, \mathbb{C}^{k}\right)$, then $\left\{A u_{j}\right\}$ is, by Lemma 6, a Cauchy sequence in $H^{-1 / 2}\left(\mathscr{D}, \mathbb{C}^{l}\right)$. Let $f$ be the limit of $\left\{A u_{j}\right\}$ in $H^{-1 / 2}\left(\mathscr{D}, \mathbb{C}^{l}\right)$. It is immaterial which sequence $\left\{u_{j}\right\}$ we choose to define $f$, and so we may set $A u=f$. Substituting $u_{j}$ into the estimate of Lemma 6 and letting $j \rightarrow \infty$, we deduce that this estimate actually holds for all $u \in \mathscr{H}^{1 / 2}\left(\mathscr{D}, \mathbb{C}^{k}\right)$. Thus, for each $u=\left(u_{i}, u_{b}\right)$ in $\mathscr{H}^{1 / 2}\left(\mathscr{D}, \mathbb{C}^{k}\right)$, the element $A u$ is defined in $H^{-1 / 2}\left(\mathscr{D}, \mathbb{C}^{l}\right)$, and the mapping $u \mapsto A u$ is continuous in the 
corresponding norms. A passage to the limit similar to the previous implies that $A u=f$ holds for $u \in \mathscr{H}^{1 / 2}\left(\mathscr{D}, \mathbb{C}^{k}\right)$ and $f \in H^{-1 / 2}\left(\mathscr{D}, \mathbb{C}^{l}\right)$ if and only if the couple $\left(u_{i}, u_{b}\right)$ satisfy the equation

$$
\begin{gathered}
\int_{\mathscr{D}}\left(u_{i}, A^{*} g\right)_{x} d x+\int_{\partial \mathscr{D}}\left(\left(B u_{b}, C^{*} g\right)_{x}-\left(C u_{b}, B^{*} g\right)_{x}\right) d s \\
=\int_{\mathscr{D}}(f, g)_{x} d x
\end{gathered}
$$

for all $g \in C^{\infty}\left(\overline{\mathscr{D}}, \mathbb{C}^{l}\right)$. In other words, Green's formula (9) is still valid for functions $u \in \mathscr{H}^{1 / 2}\left(\mathscr{D}, \mathbb{C}^{k}\right)$.

If $u \in \mathscr{H}^{1 / 2}\left(\mathscr{D}, \mathbb{C}^{k}\right)$ then $A u \in H^{-1 / 2}\left(\mathscr{D}, \mathbb{C}^{l}\right)$, and so $A u$ can be approximated by functions of $C_{\text {comp }}^{\infty}\left(\mathscr{D}, \mathbb{C}^{l}\right)$ in the $H^{-1 / 2}\left(\mathscr{D}, \mathbb{C}^{l}\right)$-norm. On multiplying $A u$ by the characteristic function $\chi_{\mathscr{D}}$ of $\mathscr{D}$ we get an element of $H^{-1 / 2}\left(\mathscr{X}, \mathbb{C}^{l}\right)$ with support in $\overline{\mathscr{D}}$. It follows that $\Phi\left(\chi_{\mathscr{D}} A u\right)$ is well defined and belongs to the local space $H_{\text {loc }}^{1 / 2}\left(\mathscr{X}, \mathbb{C}^{k}\right)$. We now show that any $u \in \mathscr{H}^{1 / 2}\left(\mathscr{D}, \mathbb{C}^{k}\right)$ can be restored through the data $A u$ and $\left.u_{b}=u\right\rceil_{\partial g}$.

To shorten notation we use the same letter $\Phi(x, y)$ for the Schwartz kernel of the pseudodifferential operator $\Phi$. Recall that $\Phi$ stands for a left fundamental solution of $A$, see Lemma 3.

Lemma 7. For each $u \in \mathscr{H}^{1 / 2}\left(\mathscr{D}, \mathbb{C}^{k}\right)$, it follows that

$$
\begin{aligned}
-\int_{\partial \mathscr{D}} & \left(\left(B u, C^{*} \Phi(x, \cdot)^{*}\right)_{y}-\left(C u, B^{*} \Phi(x, \cdot)^{*}\right)_{y}\right) d s \\
& +\int_{\mathscr{D}}\left(A u, \Phi(x, \cdot)^{*}\right)_{y} d y \\
= & \begin{cases}u(x), & \text { if } x \in \mathscr{D}, \\
0, & \text { if } x \in \mathscr{X} \backslash \bar{D} .\end{cases}
\end{aligned}
$$

Proof. The proof of Lemma 4 shows that formula (21) is actually equivalent to the equality

$$
-\Phi([\partial \mathscr{D}] \sigma u)+\Phi\left(\chi_{\mathscr{D}} A u\right)=\chi_{\mathscr{D}} u
$$

in the sense of distributions on $\mathscr{X}$, where $[\partial \mathscr{D}]$ is the surface layer on $\partial \mathscr{D}$. This follows in turn from the Green formula and the fact that $\Phi$ is a left fundamental solution of $A$ in $\mathscr{X}$, for

$$
\begin{aligned}
\Phi([\partial \mathscr{D}] \sigma u) & =\Phi\left(\chi_{\mathscr{D}} A u-A\left(\chi_{\mathscr{D}} u\right)\right) \\
& =\Phi\left(\chi_{\mathscr{D}} A u\right)-\chi_{\mathscr{D}} u,
\end{aligned}
$$

as desired.

\section{Operator-Theoretic Foundations}

The operator-theoretic foundations of the method of FischerRiesz equations are elaborated in [7, Section 11.1]. It goes back at least as far as [9]. In this section we adapt this method for study of boundary value problem (7) in the Hilbert space $\mathscr{H}^{1 / 2}\left(\mathscr{D}, \mathbb{C}^{k}\right)$.
Set

$$
\begin{gathered}
H_{1}=\mathscr{H}^{1 / 2}\left(\mathscr{D}, \mathbb{C}^{k}\right), \\
H=H_{2} \oplus L^{2}\left(\partial \mathscr{D}, \mathbb{C}^{k-k^{\prime}}\right),
\end{gathered}
$$

where $H_{2}=H^{-1 / 2}\left(\mathscr{D}, \mathbb{C}^{l}\right) \oplus L^{2}\left(\partial \mathscr{D}, \mathbb{C}^{k^{\prime}}\right)$.

Consider the mapping $M: H_{1} \rightarrow H$ given by $M u=$ $(\mathrm{Au}, \mathrm{Bu}, \mathrm{Cu})$, which corresponds to the Cauchy problem for solutions of $A u=f$ in $\mathscr{D}$ with Cauchy data $B u=u_{0}$ and $C u=$ $u_{1}$ on $\partial \mathscr{D}$. By what mentioned previously, $M$ is continuous. In Section 6 we will prove that $M$ has closed range.

Denote by $M^{*}: H \rightarrow H_{1}$ the operator that is adjoint to $M: H_{1} \rightarrow H$ in the sense of Hilbert spaces.

Lemma 8. The null-space $\operatorname{ker} M^{*}$ of the operator $M^{*}$ is separable in the topology induced from $H$.

Proof. This is true by the school fact that any subspace of a separable metric space is separable.

By $\delta_{A^{*}}(\bar{D})$ we denote the space of all infinitely differentiable solutions of the formal adjoint system $A^{*} g=0$ in a neighbourhood of the closure of $\mathscr{D}$.

Lemma 9. Assume that $g \in \mathcal{S}_{A^{*}}(\overline{\mathscr{D}})$. Then the couple ( $g \ominus$ $C^{*} g, B^{*} g$ ) belongs to ker $M^{*}$.

Proof. One has to show that $\left(M u,\left(g \ominus C^{*} g, B^{*} g\right)\right)_{H}=0$ for all $u \in H_{1}$. By the Green formula, we get

$$
\begin{aligned}
(M u & \left.,\left(g \ominus C^{*} g, B^{*} g\right)\right)_{H} \\
& =\int_{\mathscr{D}}(A u, g)_{x} d x-\int_{\partial \mathscr{D}}\left(\left(B u, C^{*} g\right)_{x}-\left(C u, B^{*} g\right)_{x}\right) d s \\
& =\int_{\mathscr{D}}\left(u, A^{*} g\right)_{x} d x \\
& =0
\end{aligned}
$$

as desired.

The subspace of $\operatorname{ker} M^{*}$ consisting of all elements of the form $\left(g \ominus C^{*} g, B^{*} g\right)$, where $g \in \mathcal{S}_{A^{*}}(\overline{\mathscr{D}})$, is separable. Hence, there are many ways to choose a sequence $\left\{g_{i}\right\}_{i=1,2, \ldots}$ in $\mathcal{S}_{A^{*}}(\bar{D})$, such that the system $\left\{\left(g_{i} \ominus C^{*} g_{i}, B^{*} g_{i}\right)\right\}$ is complete in this subspace.

In Example 12 we will show some explicit sequences $\left\{g_{i}\right\}$ with this property. For the moment we fix one of such sequences.

Lemma 10. As defined above, the system $\left\{\left(g_{i} \ominus C^{*} g_{i}, B^{*} g_{i}\right)\right\}_{i=1,2, \ldots}$ is complete in $\operatorname{ker} M^{*}$.

Proof. Let $\mathscr{F}$ be a continuous linear functional on $\operatorname{ker} M^{*}$ vanishing on each element of the system $\left\{\left(g_{i} \ominus C^{*} g_{i}, B^{*} g_{i}\right)\right\}$. Since ker $M^{*}$ is a closed subspace of $H$, the Riesz representation theorem implies the existence of an element $\left(f, u_{0}, u_{1}\right) \epsilon$ 
$\operatorname{ker} M^{*}$, such that the action of $\mathscr{F}$ on $\operatorname{ker} M^{*}$ consists in scalar multiplication with the element $\left(f, u_{0}, u_{1}\right)$. In particular,

$$
\begin{aligned}
\mathscr{F}\left(g_{i} \ominus C^{*} g_{i}, B^{*} g_{i}\right)= & \int_{\mathscr{D}}\left(g_{i}, f\right)_{x} d x \\
& -\int_{\partial \mathscr{D}}\left(\left(C^{*} g_{i}, u_{0}\right)_{x}-\left(B^{*} g_{i}, u_{1}\right)_{x}\right) d s \\
= & 0
\end{aligned}
$$

for all $i=1,2, \ldots$. Since the system $\left\{\left(g_{i} \ominus C^{*} g_{i}, B^{*} g_{i}\right)\right\}_{i=1,2, \ldots}$ is dense in the subspace of $\operatorname{ker} M^{*}$ consisting of all elements of the form $\left(g \ominus C^{*} g, B^{*} g\right)$, where $g \in \mathcal{S}_{A^{*}}(\overline{\mathscr{D}})$, we get

$$
\int_{\partial \mathscr{D}}\left(\left(u_{0}, C^{*} g\right)_{x}-\left(u_{1}, B^{*} g\right)_{x}\right) d s=\int_{\mathscr{D}}(f, g)_{x} d x
$$

for all $g \in \mathcal{S}_{A^{*}}(\overline{\mathscr{D}})$. We now use Theorem 13 which says that there exists a function $u \in \mathscr{H}^{1 / 2}\left(\mathscr{D}, \mathbb{C}^{k}\right)$, such that $A u=f$ in $\mathscr{D}$ and $B u=u_{0}, C u=u_{1}$ at the boundary of $\mathscr{D}$. In other words, $\left(f, u_{0}, u_{1}\right)=M u$. Hence it follows that $\mathscr{F}(h)=$ $(h, M u)_{H}=0$ for all $h \in \operatorname{ker} M^{*}$. Thus, $\mathscr{F} \equiv 0$, and the standard application of the Hahn-Banach theorem completes the proof.

Write $P$ for the orthogonal projection of $H$ onto its direct summand $H_{2}$. The composition $P M=(A, B)$ acting from $H_{1}$ to $H_{2}$ just amounts to the operator of boundary value problem (7) in the updated setting. More precisely, given any $f \in$ $H^{-1 / 2}\left(\mathscr{D}, \mathbb{C}^{l}\right)$ and $u_{0} \in L^{2}\left(\partial \mathscr{D}, \mathbb{C}^{k^{\prime}}\right)$, find $u \in \mathscr{H}^{1 / 2}\left(\mathscr{D}, \mathbb{C}^{k}\right)$ satisfying $A u=f$ in $\mathscr{D}$ and $B u=u_{0}$ at $\partial \mathscr{D}$. The following lemma expresses the most important property of system $\left\{g_{i}\right\}$.

Lemma 11. The system $\left\{B^{*} g_{i}\right\}_{i=1,2, \ldots}$ is complete in $L^{2}(\partial \mathscr{D}$, $\left.\mathbb{C}^{k-k^{\prime}}\right)$ if and only if PM is injective.

Proof. By the Hahn-Banach theorem, $\left\{B^{*} g_{i}\right\}$ is complete in $L^{2}\left(\partial \mathscr{D}, \mathbb{C}^{k-k^{\prime}}\right)$ if and only if any continuous linear functional $\mathscr{F}$ on $L^{2}\left(\partial \mathscr{D}, \mathbb{C}^{k-k^{\prime}}\right)$ vanishing on each element of the system is zero. Pick such a functional $\mathscr{F}$. By the Riesz representation theorem there is a function $u_{1} \in L^{2}\left(\partial \mathscr{D}, \mathbb{C}^{k-k^{\prime}}\right)$, such that $\mathscr{F}(h)=\left(h, u_{1}\right)$ for all $h \in L^{2}\left(\partial \mathscr{D}, \mathbb{C}^{k-k^{\prime}}\right)$. So we get

$$
\begin{aligned}
\left(\left(0,0, u_{1}\right),\left(g_{i} \ominus C^{*} g_{i}, B^{*} g_{i}\right)\right)_{H} & =\overline{\left(B^{*} g_{i}, u_{1}\right)_{L^{2}\left(\partial \mathscr{D}, \mathbb{C}^{k-k^{\prime}}\right)}} \\
& =\overline{\mathscr{F}\left(B^{*} g_{i}\right)} \\
& =0
\end{aligned}
$$

for all $i=1,2, \ldots$. Applying Lemma 10 we deduce that the element $\left(0,0, u_{1}\right)$ belongs to the orthogonal complement of the subspace $\operatorname{ker} M^{*}$ in $H$. Since the operator $M$ has closed range, the orthogonal complement of $\operatorname{ker} M^{*}$ coincides with the range of $M$. Hence, there is a function $u \in \mathscr{H}^{1 / 2}\left(\mathscr{D}, \mathbb{C}^{k}\right)$ satisfying $A u=0$ in $\mathscr{D}$ and $B u=0, C u=u_{1}$ at $\partial \mathscr{D}$. If the operator $P M$ is injective, then $u=0$ whence $u_{1}=0$, and $\mathscr{F}=0$. Conversely, if the functional $\mathscr{F}$ is different from zero, then $u_{1}$ is not zero and so PM fails to be injective, which is precisely the desired conclusion.
After removing the elements which are linear combinations of the previous ones from the system $\left\{B^{*} g_{i}\right\}_{i=1,2, \ldots}$, we get a sequence $\left\{g_{i_{n}}\right\}$ in $\mathcal{S}_{A^{*}}(\overline{\mathscr{D}})$, such that the system $\left\{B^{*} g_{i_{n}}\right\}$ is linearly independent. Applying then the Gram-Schmidt orthogonalisation to the system $\left\{B^{*} g_{i_{n}}\right\}$ in $L^{2}\left(\partial \mathscr{D}, \mathbb{C}^{k-k^{\prime}}\right)$, we obtain a new system $\left\{e_{n}\right\}_{n=1,2, \ldots}$ in $\mathcal{S}_{A^{*}}(\overline{\mathscr{D}})$, such that $\left\{B^{*} e_{n}\right\}$ is an orthonormal system in the space $L^{2}\left(\partial \mathscr{D}, \mathbb{C}^{k-k^{\prime}}\right)$. Moreover, $\left\{B^{*} e_{n}\right\}$ is an orthonormal basis in $L^{2}\left(\partial \mathscr{D}, \mathbb{C}^{k-k^{\prime}}\right)$, provided that $P M$ is injective. Note that the elements $e_{n}$ of the new system have explicit expressions through the elements $\left\{g_{i_{1}}, \ldots, g_{i_{n}}\right\}$ of the old system in the form of Gram's determinants.

Example 12. Assume that $A$ has real analytic coefficients outside the closure of $\mathscr{D}$ in $\mathscr{X}$. Then a familiar trick with the Laplacian $A^{*} A$ shows that $A$ has a left fundamental solution $\Phi$ whose Schwartz kernel is real analytic away from the diagonal of $(\mathscr{X} \backslash \overline{\mathscr{D}}) \times(\mathscr{X} \backslash \overline{\mathscr{D}})$. Let $\left\{x_{i}\right\}$ be a finite or countable set of points in $\mathscr{X} \backslash \overline{\mathscr{D}}$, such that each connected component of $\mathscr{X} \backslash \overline{\mathscr{D}}$ contains at least one point $x_{i}$. Then the columns of $D_{x}^{\alpha} \Phi\left(x_{i}, \cdot\right)^{*}$ belong to $\mathcal{S}_{A^{*}}(\overline{\mathscr{D}})$, and the system $\left\{B^{*} D_{x}^{\alpha} \Phi\left(x_{i}, \cdot\right)^{*}\right\}$ is complete in the subspace of $L^{2}\left(\mathscr{D}, \mathbb{C}^{k-k^{\prime}}\right)$ formed by elements of the type $\left\{B^{*} g\right\}$ with $g \in \mathcal{S}_{A^{*}}(\overline{\mathscr{D}})$.

The proof of this fact actually repeats the reasoning of Example 11.4.14 in [7]. Apparently the system of Example 12 is most convenient for numerical simulations.

\section{The Cauchy Problem}

The Green formula (9) displays the Cauchy data of $u \in$ $H^{1}\left(\mathscr{D}, \mathbb{C}^{k}\right)$ at the boundary of $\mathscr{D}$ with respect to the operator $A$. These are $B u$ and $C u$ at $\partial \mathscr{D}$. Hence we formulate the Cauchy problem as follows: given any $f \in H^{-1 / 2}\left(\mathscr{D}, \mathbb{C}^{l}\right) u_{0} \in$ $L^{2}\left(\partial \mathscr{D}, \mathbb{C}^{k^{\prime}}\right)$, and $u_{1} \in L^{2}\left(\partial \mathscr{D}, \mathbb{C}^{k-k^{\prime}}\right)$, find a function $u \in$ $\mathscr{H}^{1 / 2}\left(\mathscr{D}, \mathbb{C}^{k}\right)$, satisfying $A u=f$ in $\mathscr{D}$ and

$$
\begin{aligned}
& B u=u_{0}, \\
& C u=u_{1}
\end{aligned}
$$

at $\partial \mathscr{D}$.

The Cauchy problem for solutions of systems with injective symbol and data on the whole boundary was intensively studied in the 1960s. This study was motivated to a certain extent by [20]. For a recent account of the theory we refer to $[21,22]$.

Theorem 13. Let $f \in H^{-1 / 2}\left(\mathscr{D}, \mathbb{C}^{l}\right), u_{0} \in L^{2}\left(\partial \mathscr{D}, \mathbb{C}^{k^{\prime}}\right)$, and $u_{1} \in L^{2}\left(\partial \mathscr{D}, \mathbb{C}^{k-k^{\prime}}\right)$ be given functions. In order that there might exist a solution $u \in \mathscr{H}^{1 / 2}\left(\mathscr{D}, \mathbb{C}^{k}\right)$ to the system $A u=f$ in $\mathscr{D}$ that is subject to boundary conditions (29), it is necessary and sufficient that

$$
\int_{\partial \mathscr{D}}\left(\left(u_{0}, C^{*} g\right)_{x}-\left(u_{1}, B^{*} g\right)_{x}\right) d s=\int_{\mathscr{D}}(f, g)_{x} d x
$$

for all $g \in \mathcal{S}_{A^{*}}(\overline{\mathscr{D}})$. 
Proof. Necessity. If $u \in \mathscr{H}^{1 / 2}\left(\mathscr{D}, \mathbb{C}^{k}\right)$ is a solution of the Cauchy problem with data $f, u_{0}$, and $u_{1}$, then by the Green formula

$$
\begin{aligned}
\int_{\partial \mathscr{D}} & \left(\left(u_{0}, C^{*} g\right)_{x}-\left(u_{1}, B^{*} g\right)_{x}\right) d s \\
& =\int_{\partial \mathscr{D}}\left(\left(B u, C^{*} g\right)_{x}-\left(C u, B^{*} g\right)_{x}\right) d s \\
& =\int_{\mathscr{D}}\left((A u, g)_{x}-\left(u, A^{*} g\right)_{x}\right) d x \\
& =\int_{\mathscr{D}}(f, g)_{x} d x
\end{aligned}
$$

for all $g \in \mathcal{S}_{A^{*}}(\overline{\mathscr{D}})$, as required.

Sufficiency. We introduce a function $U$ in $\mathscr{X} \backslash \partial \mathscr{D}$ with values in $\mathbb{C}^{k}$ by the Green-type integral as follows:

$$
\begin{aligned}
U(x)= & -\int_{\partial \mathscr{D}}\left(\left(u_{0}, C^{*} \Phi(x, \cdot)^{*}\right)_{y}-\left(u_{1}, B^{*} \Phi(x, \cdot)^{*}\right)_{y}\right) d s \\
& +\int_{\mathscr{D}}\left(f, \Phi(x, \cdot)^{*}\right)_{y} d y,
\end{aligned}
$$

where $x \in \mathscr{X} \backslash \partial \mathscr{D}$. An easy calculation using (13) shows that

$$
\left(u_{0}, C^{*} \Phi(x, \cdot)^{*}\right)_{y}-\left(u_{1}, B^{*} \Phi(x, \cdot)^{*}\right)_{y}=\Phi(x, \cdot) \sigma u_{b}
$$

on $\partial \mathscr{D}$, where

$$
u_{b}=T^{-1}\left(\begin{array}{l}
u_{0} \\
u_{1}
\end{array}\right)
$$

It is clear that $u_{b}$ is of class $L^{2}\left(\partial \mathscr{D}, \mathbb{C}^{k}\right)$ if and only if $u_{0} \in$ $L^{2}\left(\partial \mathscr{D}, \mathbb{C}^{k^{\prime}}\right)$ and $u_{1} \in L^{2}\left(\partial \mathscr{D}, \mathbb{C}^{k-k^{\prime}}\right)$. Thus, formula (32) reduces to

$$
U=-\Phi\left([\partial \mathscr{D}] \sigma u_{b}\right)+\Phi\left(\chi_{\mathscr{D}} f\right)
$$

in $\mathscr{X} \backslash \partial \mathscr{D}$.

For each fixed $x \in \mathscr{X} \backslash \overline{\mathscr{D}}$, the columns of the matrix $\Phi(x, \cdot)^{*}$ belong to $\mathcal{S}_{A^{*}}(\overline{\mathscr{D}})$. Hence, (30) implies that $U$ vanishes in the complement of $\overline{\mathscr{D}}$.

Set $u=U\left\lceil_{\mathscr{D}}\right.$. We next prove that $u$ is the desired solution of the Cauchy problem. This is equivalent to saying that $u \in$ $\mathscr{H}^{1 / 2}\left(\mathscr{D}, \mathbb{C}^{k}\right), A u=f$ in $\mathscr{D}$, and $u \Gamma_{\partial \mathscr{D}}=u_{b}$ at $\partial \mathscr{D}$.

By Lemma 3.2 of [22], the double layer potential $\Phi\left([\partial \mathscr{D}] \sigma u_{b}\right)$ in $\mathscr{D}$ belongs to $\mathscr{H}^{1 / 2}\left(\mathscr{D}, \mathbb{C}^{k}\right)$. Moreover, the volume potential $\Phi\left(\chi_{\mathscr{D}} f\right)$ in $\mathscr{D}$ is of class $\mathscr{H}^{1 / 2}\left(\mathscr{D}, \mathbb{C}^{k}\right)$, which is due to Lemma 6 ibid. Hence it follows that $u \in$ $\mathscr{H}^{1 / 2}\left(\mathscr{D}, \mathbb{C}^{k}\right)$. Be the function $f$ zero, we would be able to deduce the rest of the proof from Theorem 10.3.4 of [7].

In the general case we complete $\Phi$ to a fundamental solution at step 0 of a compatibility complex of $A$, cf. Theorem 4.4 .3 of [23]. An easy computation using solvability condition (30) yields

$$
A U=-[\partial \mathscr{D}] \sigma u_{b}+\chi_{\mathscr{D}} f
$$

in the sense of distributions in $\mathscr{X}$. In particular, $A u=f$ in $\mathscr{D}$.
Since $u_{b} \in L^{2}\left(\partial \mathscr{D}, \mathbb{C}^{k}\right)$, the jump of the double layer potential $\Phi\left([\partial \mathscr{D}] \sigma u_{b}\right)$ under crossing the surface $\partial \mathscr{D}$ from $\mathscr{X} \backslash \overline{\mathscr{D}}$ to $\mathscr{D}$ just amounts to $u_{b}$. This is true even for all distributions $u_{b}$ on $\partial \mathscr{D}$ taking their values in $\mathbb{C}^{k}$, see Theorem 10.1.5 in [7]. For the square integrable densities $u_{b}$ the jump is understood in an appropriate sense including the $L^{2}\left(\partial \mathscr{D}, \mathbb{C}^{k}\right)$-norm

On the other hand, the volume potential $\Phi\left(\chi_{\mathscr{D}} f\right)$ has no jump at the boundary of $\mathscr{D}$, for $\chi_{\mathscr{D}} f \in H_{\bar{D}}^{-1 / 2}\left(\mathscr{X}, \mathbb{C}^{k}\right)$. Summarising, we conclude that $u \uparrow_{\partial \mathscr{D}}=u_{b}$, for $U$ vanishes in $\mathscr{X} \backslash \overline{\mathscr{D}}$. For a thorough treatment of this equality we refer the reader to Theorem 4.3 of [22].

\section{The Fischer-Riesz Equations}

Let $\left\{g_{i}\right\}_{i=1,2, \ldots}$ be an arbitrary sequence in $\mathcal{S}_{A^{*}}(\overline{\mathscr{D}})$ with the property that the system $\left\{\left(g_{i} \ominus C^{*} g_{i}, B^{*} g_{i}\right)\right\}$ is complete in ker $M^{*}$. Applying the Gram-Schmidt orthogonalisation to the system $\left\{B^{*} g_{i}\right\}$ in $L^{2}\left(\partial \mathscr{D}, \mathbb{C}^{k-k^{\prime}}\right)$, we obtain a new system $\left\{e_{n}\right\}_{n=1,2, \ldots}$ in $\mathcal{S}_{A^{*}}(\overline{\mathscr{D}})$, such that the system $\left\{B^{*} e_{n}\right\}$ is orthonormal in the space $L^{2}\left(\partial \mathscr{D}, \mathbb{C}^{k-k^{\prime}}\right)$.

Given any $u_{1} \in L^{2}\left(\partial \mathscr{D}, \mathbb{C}^{k-k^{\prime}}\right)$, we denote by $k_{n}\left(u_{1}\right)$ the Fourier coefficients of $u_{1}$ with respect to the system $\left\{B^{*} e_{n}\right\}$; that is,

$$
k_{n}\left(u_{1}\right)=\int_{\partial \mathscr{D}}\left(u_{1}, B^{*} e_{n}\right)_{y} d s
$$

for $n=1,2, \ldots$.

Lemma 14. If $u \in \mathscr{H}^{1 / 2}\left(\partial \mathscr{D}, \mathbb{C}^{k}\right)$, then

$$
k_{n}(C u)=\int_{\partial \mathscr{D}}\left(B u, C^{*} e_{n}\right)_{y} d s-\int_{\mathscr{D}}\left(A u, e_{n}\right)_{y} d y,
$$

where $n=1,2, \ldots$.

Proof. Using Lemma 9, we obtain

$$
\begin{aligned}
k_{n}(C u) & =\int_{\partial \mathscr{D}}\left(C u, B^{*} e_{n}\right)_{y} d s-\left(M u,\left(e_{n} \ominus C^{*} e_{n}, B^{*} e_{n}\right)\right)_{H} \\
& =\int_{\partial \mathscr{D}}\left(B u, C^{*} e_{n}\right)_{y} d s-\int_{\mathscr{D}}\left(A u, e_{n}\right)_{y} d y
\end{aligned}
$$

as desired.

Thus, in order to find the Fourier coefficients of the data $\mathrm{Cu}$ on the boundary with respect to the system $\left\{B^{*} e_{n}\right\}$ in $L^{2}\left(\partial \mathscr{D}, \mathbb{C}^{k-k^{\prime}}\right)$, it suffices to know only the data $A u$ and $B u$ of problem (7).

Theorem 15. Let $f \in H^{-1 / 2}\left(\mathscr{D}, \mathbb{C}^{l}\right)$ and $u_{0} \in L^{2}\left(\partial \mathscr{D}, \mathbb{C}^{k^{\prime}}\right)$. In order that there is a $u \in \mathscr{H}^{1 / 2}\left(\mathscr{D}, \mathbb{C}^{k}\right)$, such that $A u=f$ in $\mathscr{D}$ and $B u=u_{0}$ at $\partial \mathscr{D}$, and it is necessary and sufficient that

(1) $\sum_{n=1}^{\infty}\left|c_{n}\right|^{2}<\infty$, where $c_{n}=\int_{\partial \mathscr{D}}\left(u_{0}, C^{*} e_{n}\right)_{y} d s-$ $\int_{\mathscr{D}}\left(f, e_{n}\right)_{y} d y$, 
(2) $\int_{\partial \mathscr{D}}\left(u_{0}, C^{*} g\right)_{y} d s-\int_{\mathscr{D}}(f, g)_{y} d y=0$ for all $g \in$ $\mathcal{S}_{A^{*}}(\bar{D})$ satisfying $B^{*} g=0$ at the boundary.

Proof. Necessity. Suppose there is a function $u \in \mathscr{H}^{1 / 2}\left(\mathscr{D}, \mathbb{C}^{k}\right)$ satisfying $A u=f$ in $\mathscr{D}$ and $B u=u_{0}$ at $\partial \mathscr{D}$. Then $c_{n}=k_{n}(C u)$ for all $n=1,2, \ldots$, which is due to Lemma 14. Applying the Bessel inequality yields

$$
\sum_{n=1}^{\infty}\left|c_{n}\right|^{2}=\sum_{n=1}^{\infty}\left|k_{n}(C u)\right|^{2} \leq \int_{\partial \mathscr{D}}|C u|^{2} d s<\infty,
$$

and (1) is proved. On the other hand, (2) follows immediately from the Green formula, as desired.

Sufficiency. We now assume that (1) and (2) are satisfied. Condition (1) implies, by the Fischer-Riesz theorem, that there exists a function $u_{1} \in L^{2}\left(\partial \mathscr{D}, \mathbb{C}^{k-k^{\prime}}\right)$, such that $\left\{c_{n}\right\}_{n=1,2, \ldots}$ are the Fourier coefficients of $u_{1}$ with respect to the orthonormal system $\left\{B^{*} e_{n}\right\}$ in $L^{2}\left(\partial \mathscr{D}, \mathbb{C}^{k-k^{\prime}}\right)$. In other words, we get $c_{n}=k_{n}\left(u_{1}\right)$ for all $n=1,2, \ldots$. On substituting formulas for $c_{n}$ from (1) to these equalities we arrive at the orthogonality relations

$$
\int_{\mathscr{D}}\left(f, e_{n}\right)_{y} d y-\int_{\partial \mathscr{D}}\left(\left(u_{0}, C^{*} e_{n}\right)_{y}-\left(u_{1}, B^{*} e_{n}\right)_{y}\right) d s=0
$$

for $n=1,2, \ldots$, cf. (30).

Our next goal is to prove that the element $\left(f, u_{0}, u_{1}\right) \in$ $H$ is actually orthogonal to all elements of the system $\left\{\left(g_{i} \ominus C^{*} g_{i}, B^{*} g_{i}\right)\right\}_{i=1,2, \ldots}$ in $H$, this latter being complete in $\operatorname{ker} M^{*}$. To do this, let us recall how the system $\left\{e_{n}\right\}$ has been obtained from the system $\left\{g_{i}\right\}$.

Even if the system $\left\{\left(g_{i} \ominus C^{*} g_{i}, B^{*} g_{i}\right)\right\}$ is linearly independent in $H$, the system $\left\{B^{*} g_{i}\right\}$ may have elements which are linear combinations of the previous ones in the space $L^{2}\left(\partial \mathscr{D}, \mathbb{C}^{k-k^{\prime}}\right)$. Such elements should be eliminated from the system before applying the Gram-Schmidt orthogonalisation.

For example, suppose that, for some $i$, the equality

$$
B^{*} g_{i}=\sum_{j=1}^{i-1} c_{i, j} B^{*} g_{j}
$$

is fulfilled with suitable complex numbers $c_{i, j}$. Consider the function

$$
g_{i}^{\prime}=g_{i}-\sum_{j=1}^{i-1} c_{i, j} g_{j}
$$

which belongs to $\mathcal{S}_{A^{*}}(\overline{\mathscr{D}})$. Obviously, $\left(g_{i}^{\prime} \ominus C^{*} g_{i}^{\prime}, B^{*} g_{i}^{\prime}\right)$ lies in $\operatorname{ker} M^{*}$ and satisfies $B^{*} g_{i}^{\prime}=0$. It follows that

$$
g_{i}=\sum_{j=1}^{i-1} c_{i, j} g_{j}+g_{i}^{\prime}
$$

All the other elements $\left(g_{i} \ominus C^{*} g_{i}, B^{*} g_{i}\right)$, except for the eliminated ones, are expressed, by the contents of GramSchmidt orthogonalisation, as linear combinations of the elements $\left\{\left(e_{n} \ominus C^{*} e_{n}, B^{*} e_{n}\right)\right\}_{n=1, \ldots, i}$. Thus, any element of the system $\left\{\left(g_{i} \ominus C^{*} g_{i}, B^{*} g_{i}\right)\right\}$ has a unique expression through the elements of the system $\left\{\left(e_{n} \ominus C^{*} e_{n}, B^{*} e_{n}\right)\right\}_{n=1,2, \ldots}$ in the form

$$
g_{i}=\sum_{n=1}^{i} c_{i, n} e_{n}+g_{i}^{\prime}
$$

where $g_{i}^{\prime} \in \mathcal{S}_{A^{*}}(\overline{\mathscr{D}})$ satisfies $B^{*} g_{i}^{\prime}=0$ at the boundary $\partial \mathscr{D}$.

From equalities (41) and (45) and condition (2) of the theorem it follows immediately that

$$
\begin{aligned}
& \left(\left(f, u_{0}, u_{1}\right),\left(g_{i} \ominus C^{*} g_{i}, B^{*} g_{i}\right)\right)_{H} \\
& =\sum_{n=1}^{i} c_{i, n}\left(\left(f, u_{0}, u_{1}\right),\left(e_{n} \ominus C^{*} e_{n}, B^{*} e_{n}\right)\right)_{H} \\
& \quad+\left(\left(f, u_{0}, u_{1}\right),\left(g_{i}^{\prime} \ominus C^{*} g_{i}^{\prime}, B^{*} g_{i}^{\prime}\right)\right)_{H} \\
& =0
\end{aligned}
$$

for all $i=1,2, \ldots$. Since the system $\left\{\left(g_{i} \ominus C^{*} g_{i}, B^{*} g_{i}\right)\right\}_{i=1,2, \ldots}$ is complete in $\operatorname{ker} M^{*}$, the element $\left(f, u_{0}, u_{1}\right)$ belongs to the orthogonal complement of this subspace in $H$. Using the lemma of operator kernel annihilator, we deduce that there exists a function $u \in \mathscr{H}^{1 / 2}\left(\mathscr{D}, \mathbb{C}^{k}\right)$ satisfying $M u=\left(f, u_{0}, u_{1}\right)$. In particular, $A u=f$ in $\mathscr{D}$ and $B u=u_{0}$ at $\partial \mathscr{D}$; that is, $u$ is the desired solution of boundary value problem (7).

The convergence of the series in (1) guarantees the stability of boundary value problem (7). Under this condition, the range of the mapping $P M$ is described in terms of continuous linear functionals on the space $H$, cf. (2), which is impossible in the general case.

Corollary 16. Under the hypotheses of Theorem 15, if moreover the homogeneous adjoint boundary value problem (14) has no smooth solutions in $\bar{D}$ different from zero, then for problem (7) to have a solution $u \in \mathscr{H}^{1 / 2}\left(\mathscr{D}, \mathbb{C}^{k}\right)$ it is necessary and sufficient that

$$
\sum_{n=1}^{\infty}\left|c_{n}\right|^{2}<\infty
$$

Proof. This follows immediately from Theorem 15 since condition (2) is automatically fulfilled.

\section{Regularisation of Solutions}

Note that the proof of Theorem 15 works without the assumption that the operator $P M$ in $H$ is injective. Our next objective will be to construct an approximate solution to boundary value problem (7). To this end it is natural to assume that the homogeneous boundary value problem corresponding to (7) has only zero solution in the space $\mathscr{H}^{1 / 2}\left(\mathscr{D}, \mathbb{C}^{k}\right)$; that is, the mapping $P M$ is injective. In this case the orthonormal system $\left\{B^{*} e_{n}\right\}$ is actually complete in the space $L^{2}\left(\partial \mathscr{D}, \mathbb{C}^{k-k^{\prime}}\right)$. The orthonormal bases in $L^{2}\left(\partial \mathscr{D}, \mathbb{C}^{k-k^{\prime}}\right)$ of this form are said to be special, cf. [11], [7, Section 11.3]. 
For $x \in \mathscr{X} \backslash \partial \mathscr{D}$, we denote by $k_{n}\left(B^{*} \Phi(x, \cdot)^{*}\right)$ the $k$ row whose entries are the Fourier coefficients of the columns of the $\left(\left(k-k^{\prime}\right) \times k\right)$-matrix $B^{*} \Phi(x, \cdot)^{*}$ with respect to the orthonormal basis $\left\{B^{*} e_{n}\right\}_{n=1,2, \ldots}$ in $L^{2}\left(\partial \mathscr{D}, \mathbb{C}^{k-k^{\prime}}\right)$. More precisely, we set

$$
k_{n}\left(B^{*} \Phi(x, \cdot)^{*}\right)=\int_{\partial \mathscr{D}}\left(B^{*} \Phi(x, \cdot)^{*}, B^{*} e_{n}\right)_{y} d s
$$

for $n=1,2, \ldots$.

Lemma 17. For $n=1,2, \ldots$, the coefficients $k_{n}\left(B^{*} \Phi(x, \cdot)^{*}\right)$ are infinitely differentiable functions in $\mathscr{X} \backslash \partial \mathscr{D}$ with values in $\left(\mathbb{C}^{k}\right)^{*}$.

Proof. The assertion is obvious, for the fundamental solution $\Phi(x, y)$ is $C^{\infty}$ away from the diagonal of $\mathscr{X} \times \mathscr{X}$.

Consider the following (Schwartz) kernels $R_{N}$ defined for $x \in \mathscr{X} \backslash \partial \mathscr{D}$ and $y$ in a neighbourhood of $\overline{\mathscr{D}}$ :

$$
R_{N}(x, y)=\Phi(x, y)-\sum_{n=1}^{N} k_{n}\left(B^{*} \Phi(x, \cdot)^{*}\right)^{*} e_{n}(y)^{*}
$$

where $N=1,2, \ldots$

Lemma 18. As defined above, the kernels $R_{N}$ are $C^{\infty}$ in $x \in$ $\mathscr{X} \backslash \partial \mathscr{D}$ and $y$ in a neighbourhood of $\overline{\mathscr{D}}$ except for the diagonal $\{x=y\}$, and $A^{*}(y, D) R_{N}(\cdot, y)^{*}=0$ on this set.

Proof. This follows immediately from Lemma 17 and the fact that $e_{n} \in \mathcal{S}_{A^{*}}(\overline{\mathscr{D}})$, as desired.

The sequence $\left\{R_{N}\right\}$ provides a very special approximation of the fundamental solution $\Phi$.

Lemma 19. The sequence $\left\{B^{*} R_{N}(x, \cdot)^{*}\right\}_{N=1,2, \ldots}$ converges to zero in the norm of $L^{2}\left(\partial \mathscr{D}, \mathbb{C}^{\left(k-k^{\prime}\right) \times k}\right)$ uniformly in $x$ on compact subsets of $\mathscr{X} \backslash \partial \mathscr{D}$.

Proof. In fact, we get

$$
\begin{aligned}
B^{*} R_{N}(x, \cdot)^{*} & =B^{*} \Phi(x, \cdot)^{*}-\sum_{n=1}^{N} B^{*} e_{n} k_{n}\left(B^{*} \Phi(x, \cdot)^{*}\right) \\
& =\sum_{n=N+1}^{\infty} B^{*} e_{n} k_{n}\left(B^{*} \Phi(x, \cdot)^{*}\right)
\end{aligned}
$$

for each fixed $x \in \mathscr{X} \backslash \partial \mathscr{D}$. The right-hand side of this equality is a remainder of the Fourier series of the element $B^{*} R_{N}(x, \cdot)^{*}$ with respect to the orthonormal basis $\left\{B^{*} e_{n}\right\}$ in $L^{2}\left(\partial \mathscr{D}, \mathbb{C}^{k-k^{\prime}}\right)$. Hence, it tends to zero in the $L^{2}\left(\partial \mathscr{D}, \mathbb{C}^{\left(k-k^{\prime}\right) \times k}\right)$-norm, as $N \rightarrow \infty$. This proves the first part of the lemma. The second part follows from a general remark on Fourier series, that the mapping of $\mathscr{X} \backslash \partial \mathscr{D}$ to $L^{2}\left(\partial \mathscr{D}, \mathbb{C}^{\left(k-k^{\prime}\right) \times k}\right)$ given by $x \mapsto B^{*} \Phi(x, \cdot)^{*}$ is continuous.

The convergence of the approximations allows one to reconstruct solutions $u$ of the class $\mathscr{H}^{1 / 2}\left(\mathscr{D}, \mathbb{C}^{k}\right)$ through their data $A u$ and $B u$.
Theorem 20. Every function $u \in \mathscr{H}^{1 / 2}\left(\mathscr{D}, \mathbb{C}^{k}\right)$ can be represented by the integral formula

$$
\begin{aligned}
u(x)=\lim _{N \rightarrow \infty}\left(-\int_{\partial \mathscr{D}}\right. & \left(B u, C^{*} R_{N}(x, \cdot)^{*}\right)_{y} d s \\
& \left.+\int_{\mathscr{D}}\left(A u, R_{N}(x, \cdot)^{*}\right)_{y} d y\right)
\end{aligned}
$$

for all $x \in \mathscr{D}$.

Proof. Fix a point $x \in \mathscr{D}$. Since $R_{N}(x, \cdot)^{*}$ and $\Phi(x, \cdot)^{*}$ differ by a $k$-row of smooth solutions of the system $A^{*} g=0$ in a neighbourhood of $\overline{\mathscr{D}}$, one can write by the Green formula

$$
\begin{aligned}
u(x)= & -\int_{\partial \mathscr{D}}\left(\left(B u, C^{*} R_{N}(x, \cdot)^{*}\right)_{y}-\left(C u, B^{*} R_{N}(x, \cdot)^{*}\right)_{y}\right) d s \\
& +\int_{\mathscr{D}}\left(A u, R_{N}(x, \cdot)^{*}\right)_{y} d y
\end{aligned}
$$

for any $N=1,2, \ldots$ From $u \in \mathscr{H}^{1 / 2}\left(\mathscr{D}, \mathbb{C}^{k}\right)$ we deduce that $C u \in L^{2}\left(\partial \mathscr{D}, \mathbb{C}^{k-k^{\prime}}\right)$. Hence it follows by Lemma 19 that

$$
\lim _{N \rightarrow \infty} \int_{\partial \mathscr{D}}\left(C u, B^{*} R_{N}(x, \cdot)^{*}\right)_{y} d s=0 .
$$

Thus, letting $N \rightarrow \infty$ in (52) establishes the formula.

As mentioned, several problems of mathematical physics formulas for approximate solution like that of Theorem 20 were earlier obtained by Kupradze, see [10]. In the Cauchy problem for solutions of overdetermined elliptic systems with data on an open part of the boundary an analogous formula was derived in [11]. Chapter 11 in [7] contains many explicit applications of the formula of [11]. To the best of our knowledge, Theorem 20 is new for general boundary value problems (7).

\section{Solvability of Elliptic Boundary Value Problems}

We can now return to the classical setting of boundary value problem (7) which is $H_{1}=H^{1}\left(\mathscr{D}, \mathbb{C}^{k}\right)$. Given any $u \in$ $H^{1}\left(\mathscr{D}, \mathbb{C}^{k}\right)$, both $A u$ and $B u$ are well defined in $L^{2}\left(\mathscr{D}, \mathbb{C}^{l}\right)$ and $H^{1 / 2}\left(\partial \mathscr{D}, \mathbb{C}^{k^{\prime}}\right)$, respectively. Hence, the analysis does not require any function spaces of negative smoothness but distributions. More generally, let $s$ be a natural number. Given any $f \in H^{s-1}\left(\mathscr{D}, \mathbb{C}^{l}\right)$ and $u_{0}$ in $H^{s-1 / 2}\left(\partial \mathscr{D}, \mathbb{C}^{k^{\prime}}\right)$, we look for a $u \in H^{s}\left(\mathscr{D}, \mathbb{C}^{k}\right)$ satisfying (7). Theorem 15 still applies to establish the existence of a weak solution $u \in \mathscr{H}^{1 / 2}\left(\mathscr{D}, \mathbb{C}^{k}\right)$, if the conditions (1) and (2) are fulfilled. To infer the existence of a classical solution, one needs a regularity theorem for weak solutions in $\mathscr{H}^{1 / 2}\left(\mathscr{D}, \mathbb{C}^{k}\right)$ saying that any weak solution is actually a classical one; that is, $u \in H^{s}\left(\mathscr{D}, \mathbb{C}^{k}\right)$ provided $A u \in H^{s-1}\left(\mathscr{D}, \mathbb{C}^{l}\right)$ and $B u \in H^{s-1 / 2}\left(\partial \mathscr{D}, \mathbb{C}^{k^{\prime}}\right)$. This is the case if (7) is an elliptic boundary value problem; that is, $A$ is elliptic $(l=k)$, and the pair $(A, B)$ satisfies the ShapiroLopatinskii condition at the boundary of $\mathscr{D}$, see Section 10.5 
in [19]. For general operators $A$ with injective symbol the regularity problem may be reduced to a regularity theorem for weak solutions of $A^{*} A u=A^{*} f$ in $\mathscr{D}$ with boundary data $B u=u_{0}$ and $B^{*} A u=B^{*} f$ at $\partial \mathscr{D}$, see Lemma 4 .

Corollary 21. Suppose a regularity theorem holds for (7). Let $f \in H^{s-1}\left(\mathscr{D}, \mathbb{C}^{l}\right)$ and $u_{0} \in H^{s-1 / 2}\left(\partial \mathscr{D}, \mathbb{C}^{k^{\prime}}\right)$, where $s=$ $1,2, \ldots$. Then, in order that there is a $u \in H^{s}\left(\mathscr{D}, \mathbb{C}^{k}\right)$, such that $A u=f$ in $\mathscr{D}$ and $B u=u_{0}$ at $\partial \mathscr{D}$, and it is necessary and sufficient that

(1) $\sum_{n=1}^{\infty}\left|c_{n}\right|^{2}<\infty$, where $c_{n}=\int_{\partial \mathscr{D}}\left(u_{0}, C^{*} e_{n}\right)_{y} d s-$ $\int_{\mathscr{D}}\left(f, e_{n}\right)_{y} d y$

(2) $\int_{\partial \mathscr{D}}\left(u_{0}, C^{*} g\right)_{y} d s-\int_{\mathscr{D}}(f, g)_{y} d y=0$ for all $g \in$ $\mathcal{S}_{A^{*}}(\overline{\mathscr{D}})$ satisfying $B^{*} g=0$ at the boundary.

Proof. It is sufficient to prove the sufficiency of conditions (1) and (2). If the conditions (1) and (2) are satisfied, then there exists a function $u \in \mathscr{H}^{1 / 2}\left(\mathscr{D}, \mathbb{C}^{k}\right)$, such that $A u=f$ in $\mathscr{D}$ and $B u=u_{0}$ at $\partial \mathscr{D}$. Since $A u \in H^{s-1}\left(\mathscr{D}, \mathbb{C}^{l}\right)$ and $B u \in H^{s-1 / 2}\left(\partial \mathscr{D}, \mathbb{C}^{k^{\prime}}\right)$, the regularity theorem implies that $u \in H^{s}\left(\mathscr{D}, \mathbb{C}^{k}\right)$, as desired.

If (7) is elliptic, then the problem $\left(A^{*}, B^{*}\right)$ is adjoint to (7) with respect to the Green formula. By the Fredholm property, the space of all $g \in \mathcal{S}_{A^{*}}(\overline{\mathscr{D}})$ satisfying $B^{*} g=0$ at $\partial \mathscr{D}$ is finite dimensional. Moreover, the condition (2) alone is sufficient for the existence of a solution $u \in H^{s}\left(\mathscr{D}, \mathbb{C}^{k}\right)$ to problem (7). Hence it follows that for elliptic boundary value problems the condition (1) is automatically fulfilled. In other words, if boundary value problem (7) is elliptic, then condition (2) is necessary and sufficient for a solution to exist. If moreover each $g \in \mathcal{S}_{A^{*}}(\overline{\mathscr{D}})$ satisfying $B^{*} g=0$ at the boundary vanishes, then problem (7) is solvable for all data $f \in L^{2}\left(\mathscr{D}, \mathbb{C}^{l}\right)$ and $u_{0} \in H^{1 / 2}\left(\partial \mathscr{D}, \mathbb{C}^{k^{\prime}}\right)$. Condition (1) is of purely theoretical interest, for there is no efficient way to verify it.

Thus, the regularity problem for weak solutions of (7) is still of primary character in the study of boundary value problems. On the other hand, our approach demonstrates rather strikingly that Theorem 20 is of great importance for numerical simulation.

Corollary 21 applies in particular to boundary value problems for generalised Cauchy-Riemann systems in the space [23-25], see also $[18,26]$.

\section{Acknowledgment}

The research of the first author was supported by the German Academic Exchange Service (DAAD).

\section{References}

[1] L. A. Aizenberg and A. P. Yuzhakov, Integral Representations and Residues in Many-Dimensional Complex Analysis, Nauka, Novosibirsk, Russia, 1979.
[2] L. Boutet de Monvel, "Boundary problems for pseudo-differential operators," Acta Mathematica, vol. 126, no. 1-2, pp. 11-51, 1971.

[3] L. S. Wrobel and M. H. Aliabadi, The Boundary Element Method, John Wiley \& Sons, New York, NY, USA, 2002.

[4] J. T. Katsikadelis, Boundary Elements Theory and Applications, Elsevier, Oxford, UK, 2002.

[5] W. C. Gibson, The Method of Moments in Electromagnetics, Chapman \& Hall/CRC, London, UK, 2008.

[6] G. Beer, I. Smith, and C. Duenser, The Boundary Element Method and Programming: For Engineers and Scientists, Springer, Berlin, Germany, 2008.

[7] N. N. Tarkhanov, The Cauchy Problem for Solutions of Elliptic Equations, vol. 7 of Mathematical Topics, Akademie, Berlin, Germany, 1995.

[8] M. Picone, "Nuovi metodi per il calcolo delle soluzioni delle equazioni a derivate parziali della fisica matematica," Annales Scientiques de l'Universite Jassy, vol. 26, pp. 183-232, 1940.

[9] M. Picone and G. Fichera, "Neue funktional-analytische Grundlagen für die Existenzprobleme und Lösungsmethoden von Systemen linearer partieller Differentialgleichungen," Monatshefte für Mathematik, vol. 54, no. 3, pp. 188-209, 1950.

[10] V. D. Kupradze, "Approximate solution of problems of mathematical physics," Uspekhi Matematicheskikh Nauk, vol. 22, no. 2, pp. 59-107, 1967.

[11] O. V. Karepov and N. N. Tarkhanov, "The Fischer-Riesz equations method in the ill-posed Cauchy problem for systems with injective symbols," Journal of Inverse and Ill-Posed Problems, vol. 1, no. 4, pp. 307-330, 1993.

[12] A. Douglis and L. Nirenberg, "Interior estimates for elliptic systems of partial differential equations," Communications on Pure and Applied Mathematics, vol. 8, pp. 503-538, 1955.

[13] M. H. Protter, "Overdetermined first order elliptic systems," in Maximum Principles and Eigenvalue Problems in Partial Differential Equations (Knoxville, TN, 1987), P. W. Schaefer, Ed., vol. 175 of Research Notes in Mathematics, pp. 68-81, Pitman, New York, NY, USA, 1988.

[14] L. R. Volevič, "Solubility of boundary value problems for general elliptic systems," Matematicheskiu Sbornik, vol. 68, no. 110, pp. 373-416, 1965.

[15] C. Cosner, "On the definition of ellipticity for systems of partial differential equations," Journal of Mathematical Analysis and Applications, vol. 158, no. 1, pp. 80-93, 1991.

[16] C. B. Morrey, "Second-order elliptic systems of differential equations," in Contributions to the Theory of Partial Differential Equations, vol. 33 of Annals of Mathematics Studies, pp. 101-159, Princeton University Press, Princeton, NJ, USA, 1954.

[17] K. Krupchyk, W. M. Seiler, and J. Tuomela, "Overdetermined elliptic systems," Foundations of Computational Mathematics, vol. 6, no. 3, pp. 309-351, 2006.

[18] M. S. Agranovič, "Boundary value problems for systems of first order pseudodifferential operators," Uspekhi Matematicheskikh Nauk, vol. 24, no. 1, pp. 61-125, 1969.

[19] Y. Roitberg, Elliptic Boundary Value Problems in the Spaces of Distributions, vol. 384 of Mathematics and Its Applications, Kluwer Academic, Dordrecht, The Netherlands, 1996.

[20] A. P. Calderón, "Boundary value problems for elliptic equations," in Outlines Joint Sympos. Partial Differential Equations (Novosibirsk, 1963), pp. 303-304, Academy of Sciences of the USSR Siberian Branch, Moscow, Russia, 1963. 
[21] L. R. Volevich and A. R. Shirikyan, "Stable and unstable manifolds for nonlinear elliptic equations with a parameter," Transactions of the Moscow Mathematical Society, vol. 61, pp. 97-138, 2000.

[22] I. V. Shestakov and A. A. Shlapunov, "On the Cauchy problem for operators with injective symbols in the spaces of distributions," Journal of Inverse and Ill-Posed Problems, vol. 19, no. 1, pp. 127-150, 2011.

[23] I. Stern, "Boundary value problems for generalized CauchyRiemann systems in the space," in Boundary Value and Initial Value Problems in Complex Analysis: Studies in Complex Analysis and Its Applications to Partial Differential Equations, I (Halle, 1988), vol. 256 of Pitman Research Notes in Mathematics Series, pp. 159-183, Longman Scientific and Technical, Harlow, UK, 1991.

[24] I. Stern, "On the existence of Fredholm boundary value problems for generalized Cauchy-Riemann systems in the space," Complex Variables: Theory and Application, vol. 21, no. 1-2, pp. 19-38, 1993.

[25] I. Stern, "Direct methods for generalized Cauchy-Riemann systems in the space," Complex Variables: Theory and Application, vol. 23, no. 1-2, pp. 73-100, 1993.

[26] K. O. Friedrichs and P. D. Lax, "Boundary value problems for first order operators," Communications on Pure and Applied Mathematics, vol. 18, pp. 355-388, 1965. 




Advances in

Operations Research

mansans

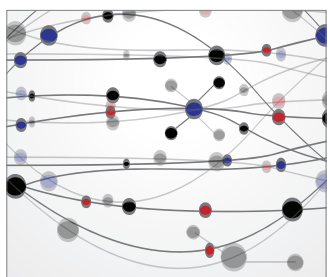

The Scientific World Journal
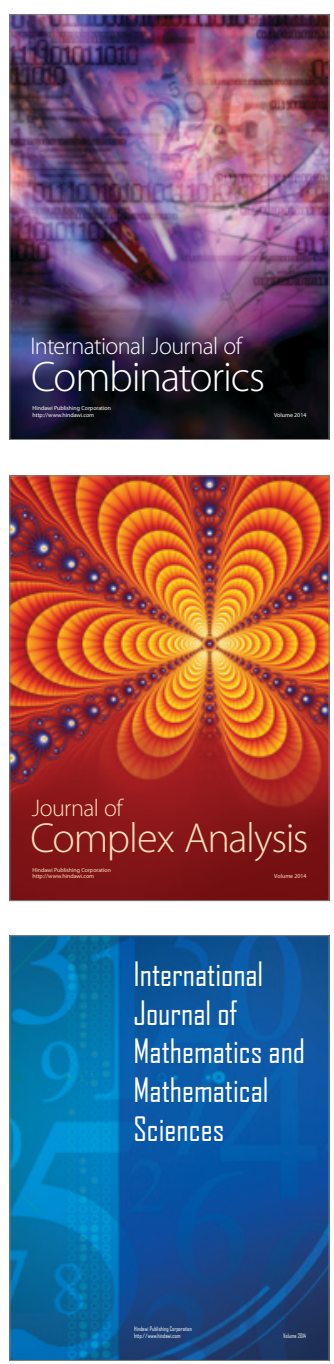
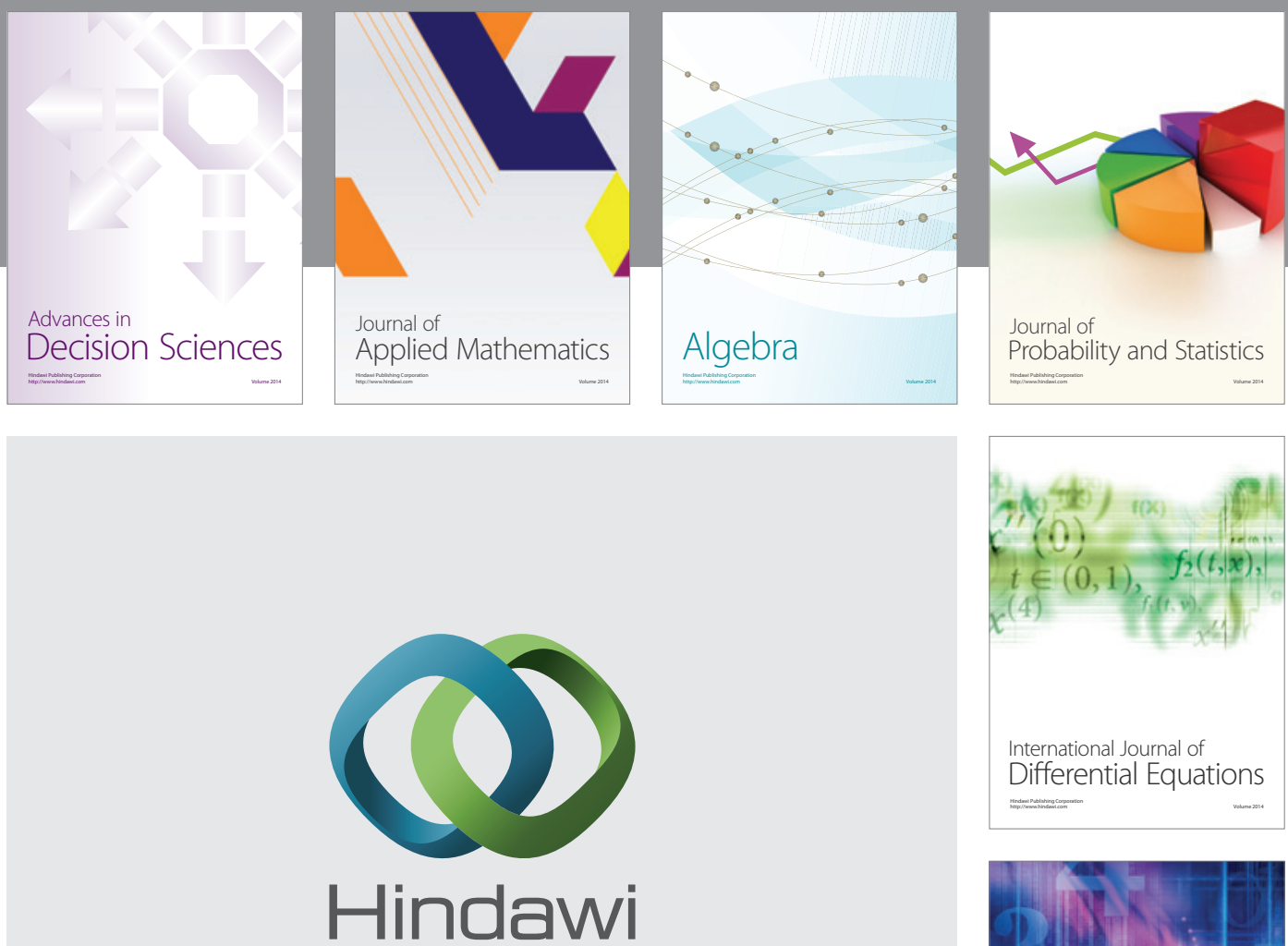

Submit your manuscripts at http://www.hindawi.com


Journal of

Function Spaces



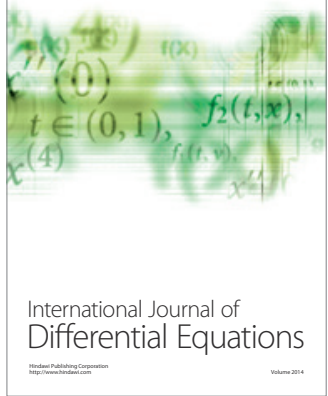
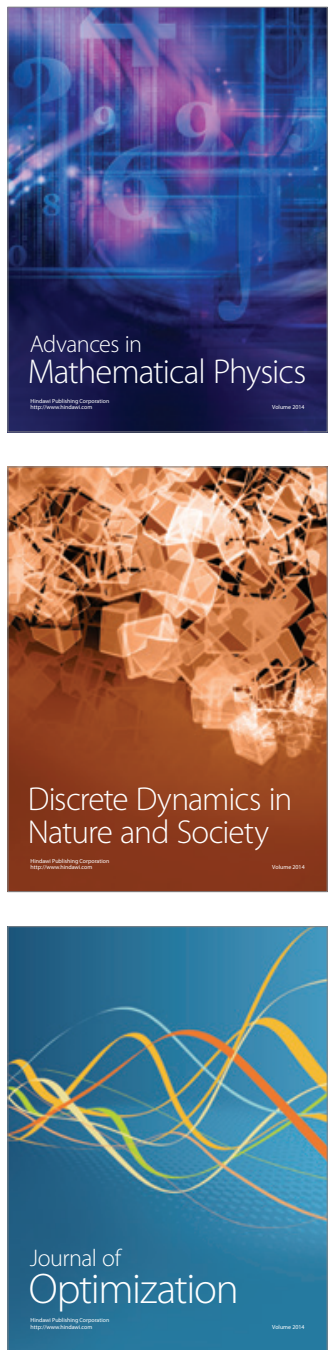